\title{
Metal Organic Framework Nano Particles: Potential Contrast Agents for Magnetic Resonance Imaging
}

\author{
Wenyan Dan*, Xiang dong Fang and Siyu Wang \\ College of Chemical Science and Engineering, Tongji University, Yangpu, China
}

Received: January 20, 2018; Published: January 31, 2018

*Corresponding author: Wenyan Dan, College of Chemical Science and Engineering, Shanghai Key Laboratory of Chemical Assessment and Sustainability (CAS), Tongji University, 1239 Siping Road, Yangpu District, Shanghai 200092, People’s Republic of China, Tel: 86-21-65982670;

Email:wydan@tongji.edu.cn

\section{Abstract}

Metal-organic framework nano-particles are a type of novel organic-inorganic hybrid materials, which has been investigated as potential contrast agents in magnetic resonance imaging (MRI). This mini review focuses on recent research progress concerning the synthesis, stability and cytotoxicityof metalorganic framework nano-particles.

Keywords: Metal-organic framework nano-particles; Contrast agents; Magnetic resonance imaging

\section{Introduction}

Metal-organic frameworks (MOFs)assembled by metal ions (or metal clusters) and organic ligandsare under the pursuit of heavy research endeavor in the areas such as catalysis [1], gas separation [2], luminescence [3], drug delivery [4], and magnetic materials [5] in past decades. In contrast tozeolites, MOF smerit from relatively facile control of their composition and structural parameters (e.g., pore sizes and shapes) by a judicious choice of corresponding metal ions and organic ligands. In addition, good biocompatibility of MOFs obtained by using bioorganic linkers such as adenine [6] and nicotinic acid [7], make themselves favorable materials of candidate in biomedicine. In general, solvothermal MOFs synthesis promoted by microwave irradiation has been utilized to achieve high yields, and more importantly, to obtain size-control of nanoparticle product [8], which further determines the chemical and physical properties of the particles. On the other hand, the delicacy of physiological systems requires the diagnostic process of diseases need to be precisely controlled in sizes. For example, parenteral treatments often choose stable a solution or suspension of nanoparticles with sizes smaller than $200 \mathrm{~nm}$ in order to circulate freely through the smallest capillary vessels. In this regard, some functional metal-organic framework nano-particles have been investigated in the diagnostics for medical purposes.

This mini review will briefly discuss the current research using metal-organic framework nano-particles as potential contrast agents (CA) in magnetic resonance imaging (MRI). MRIis an important diagnostic medical technique owing to its nature of high spatial resolution and noninvasive measurement. In fact, the contrast in MR images is derived from a complex interplay between instrument parameters and intrinsic differences in the relaxation rates of tissue water protons [9]. CA such as a $\mathrm{Gd}^{\mathrm{III}}$ chelateis generally used to increase the sensitivity of MRI by locally reducing the proton relaxation times. The magnitude of this effect on the longitudinal relaxation time T1 (or transverse relaxation time T2) is measured as the relaxivity R1 (or R2, respectively)and normalized to $1 \mathrm{mM}$ [CA] concentration at a given magnetic field strength. Maspoch reported the synthesis of a highly stable nanoscale $\mathrm{Cu}(\mathrm{II}) / \mathrm{Gd}$ (III) MOF system (CAMOF-1) and macro cyclic DOTP $(1,4,7,10$-tetraazacyclododecane-1,4,7,10tetramethylenephosphonic acid) [10], which has been investigated as a contrast agentof MRI. The nanoscale CAMOF-1 (The size of particles is less than $200 \mathrm{~nm}$ )demonstrates interesting relaxometric properties with $5 \mathrm{mM}^{-1} \cdot \mathrm{s}^{-1}$ of R1 at high field of $500 \mathrm{MHz}$ and a maximum R1 $=15 \mathrm{mM}^{-1} \bullet \mathrm{s}^{-1}$ at $40 \mathrm{MHz}$, three times higher than the corresponding relaxivity for [GdDOTP]5- at $\mathrm{pH}=7.4$. Importantly, the reported CA property of nano-scale MOFs remains stable over a wide $\mathrm{pH}$ range and increases with temperature.

The leakage of free toxic $\mathrm{Cu}$ (II) and Gd (III) ions into physiological system can be prevented by strong chelation of DOTP towards these cations in solution, and the nano-scale CAMOF-1 does not show significant toxicity in the evaluated systems. In 2017, Chen's group reported the study of zwitter ionic manganese and gadolinium MOFs as efficient CA for in vivo MRI [11]. Two 
water-stable Mn- andGd-based MOFs, $\left\{\left[\mathrm{Mn}_{2}(\mathrm{Cmdcp})_{2}\left(\mathrm{H}_{2} \mathrm{O}\right)_{2}\right] \bullet \mathrm{H}_{2} \mathrm{O}\right\}$ n (1) and $\left\{\left[\mathrm{Gd}(\mathrm{Cmdcp})-\left(\mathrm{H}_{2} \mathrm{O}\right)_{3}\right]\left(\mathrm{NO}_{3}\right) \cdot 3 \mathrm{H}_{2} \mathrm{O}\right\} \mathrm{n}$ (2) $\left(\mathrm{H}_{3} \mathrm{CmdcpBr}=\right.$ $\mathrm{N}$-(4-carboxybenzyl)-(3,5-dicarboxyl) pyridinium bromide), are hence prepared and the results of in vitro magnetic resonance imagingindicate that MOFs 1 and 2 possess relaxivity R1 values of17.50 and $13.46 \mathrm{mM}^{-1} \bullet \mathrm{S}^{-1}$, respectively, which are superior to that of Gd-DTPA (R1 $=4.87 \mathrm{mM}^{-1} \bullet \mathrm{S}^{-1}$, DTPA $=$ di ethylene tri amine penta acetate) in the control experiments.In addition, the sizes of nano-particles can be controlled(less than $100 \mathrm{~nm}$ ). Encouragingly, smaller leakage rates and less cytotoxicity of these MOFs materials indicate that compounds 1 and 2 can be further exploited as contrast agents in MRI experiments.

\section{Conclusion and Proposal for Further Research}

This short review highlights the current studies of nano scale metal-organic frameworks materials with high stability and relativity in MRI. These MOFs systems merit from small leakage rates of metal ions and low cytotoxicity and therefore become quite promising in the application of MRI contrast agents. The effect of conglomeration of nano-particles in the flow of capillary vessels has to be modeled and evaluated in the settings of physiological environments on the next stage, because nano-particles tend to aggregate and may cause severe clot problems in tissues. More studies are still needed to clarify the MRI contrast agent path of nano-scale MOFs materials before any clinical trial.

\section{References}

1. Liu J, Chen L, Cui H, Zhang J, Zhang L, et al. (2014) Applications of metal-organic frameworks in heterogeneous supramolecular catalysis. Chemical Society Reviews 43(16): 6011-6061.

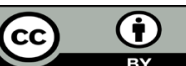

This work is licensed under Creative Commons Attribution 4.0 License

Submission Link: http://biomedres.us/submit-manuscript.php
2. Li JR, Sculley J, Zhou HC (2012) Metal-organic frameworks for separations. Chemical Reviews 112(2): 869-932.

3. Rocca JD, Liu DM, Lin WB (2011) Nanoscale metal-organic frameworks for biomedical imaging and drug delivery. Accounts of Chemical Research 44(10): 957-968.

4. Cui Y, Yue Y, Qian G, Chen B (2012) Luminescent functional metal-organic frameworks. Chemical Reviews 112(2): 1126-1162.

5. Kurmoo M (2009) Magnetic metal-organic frameworks. Chemical Society Reviews 38(5): 1353-1379.

6. An J, Fiorella RP, Geib SJ, Rosi NL (2009) Synthesis, structure, assembly, and modulation of the $\mathrm{CO}_{2}$ adsorption properties of a zincadeninatemacrocycle. Journal of the American Chemical Society 131(24): 8401-8403.

7. Miller SR, Heurtaux D, Baati T, Horcajada P, Grenèche JM, et al. (2010) Biodegradable therapeutic MOFs for the delivery of bioactive molecules. Chemical Communications 46(25): 4526-4528.

8. Chalati T, Horcajada P, Gref R, Couvreur P, Serre C (2011) Optimisation of the synthesis of MOF nanoparticles made of flexible porous iron fumarate MIL-88A. Journal of Materials Chemistry 21(7): 2220.

9. Horcajada P, Gref R, Baati T, Allan PK, Maurin G, et al. (2012) Metalorganic frameworks in biomedicine. Chemical Reviews 112(2): 12321268.

10. Carné Sánchez A, Bonnet CS, Imaz I, Lorenzo J, Tóth E, et al. (2013) Relaxometrystudies of a highly stable nanoscale metal-organic framework made of $\mathrm{Cu}$ (II), Gd (III), and the macrocyclic DOTP. Journal of the American Chemical Society 135(47): 17711-17714.

11. Qin L, Sun ZY, Cheng K, Liu SW, Pang JX, et al. (2017) Zwitter ionic manganese and gadolinium metal-organic frameworks as efficient contrast agents for in vivo magnetic resonance imaging. ACS Applied Materials \& Interfaces 9(47): 41378-41386.

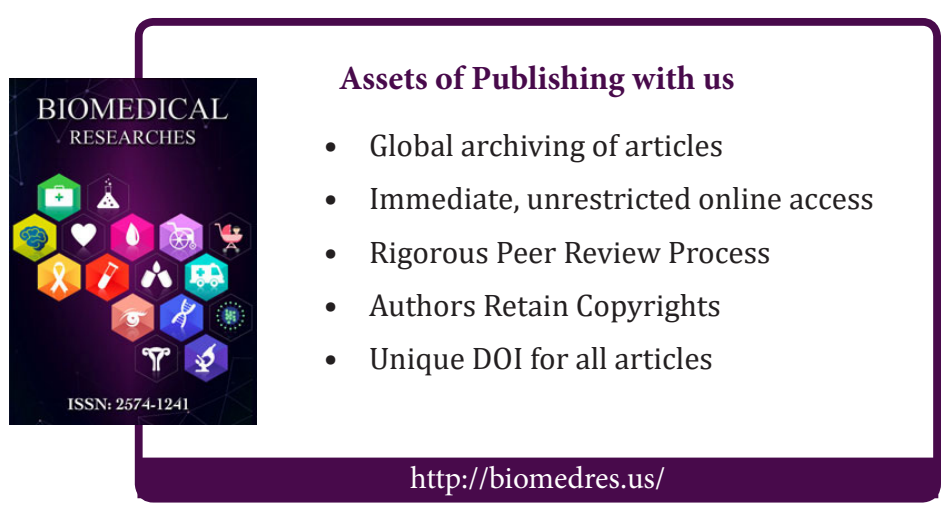

